\title{
DIFFERENCES IN PHYSIOLOGGCAL INFLUENGES ON HEART RATE RECOVERY BETWEEN TRAINED AND UNTRAINED ADULTS
}

\author{
Jennifer A. Bunn, ${ }^{1, A, B, C, D}$ Elizabeth K. Wells, ${ }^{1, C, D}$ Megan L. Avery, ${ }^{1, B, C, D}$ John P. Manor ${ }^{2, C, D}$ \\ ${ }^{1}$ Campbell University, Department of Physical Therapy, USA \\ ${ }^{2}$ Campbell University School of Osteopathic Medicine, USA \\ A Study Design; ${ }^{B}$ Data Collection; ${ }^{\mathrm{C}}$ Statistical Analysis; ${ }^{\mathrm{D}}$ Manuscript Preparation \\ Address for correspondence: \\ Jennifer Bunn \\ PO Box 1090 \\ Buies Creek, NC 27506, 919-924-2019, USA \\ E-mail:bunnj@campbell.edu
}

\begin{abstract}
Ahstract The purpose of this study was to evaluate differences in heart rate recovery ( $\mathrm{HRr}$ ) in trained and untrained adults, while assessing the role of physiological and emotional factors. Eighteen untrained and 21 trained participants completed a maximal exercise test and a 20 -min treadmill exercise at $55-70 \%$ heart rate reserve, and emotional state was assessed prior to exercise. Multiple regression was used to assess relationships between heart rate recovery and physiological and emotional assessments. The trained group had a higher relative maximal oxygen consumption $(p<0.001)$, lower resting heart rate $(p<0.001)$, and faster short- and long-term heart rate recovery $(p<0.05)$ than the untrained group. Resting heart rate was the most predictive measure with $\mathrm{HRr}$ for the trained group $(R=0.551-0.818)$, whereas resting heart rate, maximal heart rate, and fitness were predictors of recovery in the untrained group $(R=0.764-0.977)$. The results show the predominant parasympathetic influence on $\mathrm{HRr}$ in the trained group, but indicates influence of fitness and exercise intensity on recovery in the untrained group. Thus, fitness appears to influence $\mathrm{HRr}$ in those only with low fitness. This notion may help influence the behavior of untrained individuals to improve fitness to reduce risk of mortality and morbidity.
\end{abstract}

Key worlls aerobic fitness, cardiovascular, cardiorespiratory, health

\section{Introduction}

Heart rate recovery $(\mathrm{HRr})$ is a measure of parasympathetic response to exercise and is modifiable with aerobic training (Kokkinos et al., 2012; Matsuo et al., 2014). Onset of exercise increases sympathetic activation, which increases heart rate (HR) with consideration to exercise demand (Pierpont, Adabag, Yannopoulos, 2013), and parasympathetic reactivation occurs at the cessation of exercise and reduces HR back to resting levels. Although not entirely understood, the influence of parasympathetic reactivation is supported by the physiological response of plasma norepinephrine that peaks approximately one to two minute post-exercise (Pierpont et al., 2013). HRr is utilized as a prognostic indicator of mortality risk, with a delayed parasympathetic response indicating higher 
risk (Kokkinos et al., 2012). Disease, deconditioning, and body composition are believed to contribute to delayed responses, with severity of disease or deconditioning correlating to higher risk (Kokkinos et al., 2012; Matsuo et al., 2014; Pierpont et al., 2013).

Research has shown that $\mathrm{HRr}$ is predictable among healthy children (Singh, Rhodes, Gauvreau, 2008) and adults, with higher body composition and decreased aerobic capacity corresponding to slower HRr values (Brinkworth, Noakes, Buckley, Clifton, 2006; Carroll, Marshall, Ingle, Borkoles, 2012; Georgiopoulou, et al.; 2012). Body mass index (BMI), body fat, and waist circumference have been shown to be modifiable with cardiovascular (CV) exercise, and have strong correlations with $\mathrm{HRr}$ among healthy and sedentary individuals (Brinkworth et al., 2006; Carroll et al., 2012; Esco, Williford, Olson, 2011; Kim et al., 2010; Matsuo et al., 2014; Thomson et al., 2010). Essentially, $\mathrm{HRr}$ has been shown to improve in a variety of populations (including diseased and overweight/obese) with exercise participation, but the exact physiological mechanisms of influence is not well understood. Adding further confusion is the emotional or mood influence on the parasympathetic and sympathetic nervous systems (Weir, 2011). The Profile of Mood States (POMS) questionnaire was designed as a reliable and validated measure of emotional state that assesses anger, vigor, fatigue, tension, depression, and confusion as well as total mood disturbance (Bourgeois, LeUnes, Meyers, 2010; McNair, 1971). Schmikli, De Vries, Backx (2010), found that POMS was sensitive to changes in workload and recovery and correlated well with rate of perceived exertion. Using the POMS, recent literature has shown that HRr from submaximal exercise was not impacted by fitness levels or emotional state among individuals with at least average fitness (Bunn et al., 2017).

With the consistent demonstration that HRr may be modifiable with CV fitness training and changes in body composition, it's not clear to what extent the factors have influence, or what other factors may contribute to changes in HRr. As such, the primary purpose of this study was to evaluate differences in $\mathrm{HRr}$ between untrained and trained individuals after maximal and submaximal exercises while simultaneously assessing the role of physiological and emotional factors on $\mathrm{HRr}$ in both populations.

\section{Material and methods}

\section{Participants}

Prior to participation in the study, all participants provided a written informed consent as approved by the university Institutional Review Board. Eighteen untrained, overweight or obese males $(n=5)$ and females $(n=13)$ and 21 recreationally trained, normal weight males $(n=7)$ and females $(n=14)$ participated in this study. Inclusion criteria for the untrained group were: a BMI of or greater than 25, or a waist circumference of $102 \mathrm{~cm}$ or larger for men and $88 \mathrm{~cm}$ or larger for women, and self-report of a sedentary lifestyle. Inclusion criteria for the trained group were: a BMl ranging from 18.5 to 24.9 and self-report of exercise participation of at least $150 \mathrm{~min} / \mathrm{wk}$ of moderate intensity exercise or $75 \mathrm{~min} / \mathrm{wk}$ of vigorous intensity exercise. All subjects had to be between the ages of 18-55 years old, and considered low to moderate risk for CV disease without any signs or symptoms of CV disease (Riebe, Ehrman, Liguori, Magal, 2017) .

\section{Procedures}

Participants completed two testing sessions 48 hours apart. The first testing session consisted of assessing height, weight, resting hemodynamics, BMI, body composition, a psychological assessment, and a treadmill maximal 
graded exercise test (GXT). Height was measured using a standard stadiometer. Weight and body composition was measured using bioelectrical impendence analysis (BIA) using the InBody 570 (Biospace, Cerritos, CA). Resting heart rate and blood pressure were measured manually using standard procedures after the participant had been seated for at least five minutes. Participants completed the psychological assessment using the online version of the POMS questionnaire (Mackenzie, 2001). Mood states of anger, confusion, depression, fatigue, tension, vigor, and total mood disturbance were used to determine the participant's psychological state prior to exercise. These moods have been shown to be stable in various scenarios (Bourgeois et al., 2010; Mackenzie, 2001; Pronk, Crouse, Rohack, 1995). Mood scores were totaled to determine the disturbance (-32-200) of the participant, with a high positive number indicating an angry or sad mood, and a high negative number indicating a happy or excited mood (Mackenzie, 2001; Weinberg, Gould, 2011).

For the maximal GXT, the Bruce protocol was used for the untrained participants, and a modified test was used for the trained participants, wherein they ran at a challenging pace and treadmill incline was increased $2 \%$ every three minutes, until volitional exhaustion. During the GXT, oxygen consumption $\left(\mathrm{VO}_{2}\right)$ and carbon dioxide production were measured using a metabolic analyzer (Parvomedics, Sandy, UT). HR was measured via a Polar Chest Strap (Lake Success, NY), and was monitored for five minutes post-exercise. After completion of the GXT, participants completed a 2-min cool-down walking at $1.12 \mathrm{~m} \cdot \mathrm{s}^{-1}$, and then sat in a chair for three minutes to obtain five full minutes of $\mathrm{HRr}$.

The participants returned for the second testing session 48 hours after the initial session, and completed a 20 -minute treadmill exercise at $55-70 \%$ HR reserve. HR was again measured using the Polar chest strap. After completion of the 20-minute exercise, participants walked at $1.12 \mathrm{~m} \cdot \mathrm{s}^{-1}$ for two minutes and then sat in a chair for an additional three minutes. HR was monitored for the duration of the exercise and post-exercise for 5 minutes.

Absolute HRr was calculated by subtracting HR measurement post-exercise heart rates from the peak HR obtained during bout. Relative HRr was calculated by dividing the absolute HRr by peak HR and multiplying by 100 . Recovery HR measurement were taken 2- and 5-min after the GXT, and 1-, 2-, and 5-min after the submaximal bout.

\section{Statistical analysis}

Statistical analysis was done using SPSS (Chicago, IL), with an a priori alpha level set at 0.05 . Tests for normality were run prior to the parametric assessments. Multivariate analysis of variance (MANOVA) was used to evaluate group differences in $\mathrm{HRr}$, body composition, $\mathrm{BMI}$, resting hemodynamics, $\mathrm{VO}_{2}$ max, and POMS scores. Multiple step-wise regression was used to assess which variables (e.g. resting $\mathrm{HR}, \mathrm{VO}_{2} \mathrm{max}, \mathrm{BMI}$, body composition, mood states) were related to $\mathrm{HRr}$ after the maximal GXT (2- and 5-min HRr) and submaximal exercise (1-, 2-, and 5 -min HRr). Data are presented as mean \pm standard deviation (SD). Effect size of group differences was calculated using Cohen's $d$ and $95 \%$ confidence intervals. Interpretation of effect sizes was as follows: 0.2 was considered small, 0.5 was considered moderate, and 0.8 or larger was considered large (Kazis, Anderson, Meenan, 1989). 


\section{Results}

\section{Group differences}

The MANOVA revealed a significant main effect between groups $\left(\right.$ Lambda $_{9,29}=0.238, p<0.001$; Table 1). Specifically, the groups were statistically different in mass $\left(F_{1,37}=21.55, p<0.001\right)$, resting $H R\left(F_{1,37}=15.527\right.$, $p<0.001)$, percent body fat $\left(F_{1,37}=62.799, p<0.001\right)$, and BMI $\left(F_{1,37}=44.982, p<0.001\right)$. Additionally, the POMS score for vigor was found to be significantly different between groups $\left(F_{1,37}=6.754, p=0.013\right)$. Of particular note, is that the untrained group can truly be considered obese as the mean $\mathrm{BMI}$ is considered obese and the mean percent body fat would be classified as poor to very poor regardless of age and gender (Riebe et al., 2017).

Significant group differences were found at the first testing session for relative $\mathrm{VO}_{2} \max \left(\mathrm{F}_{1,37}=47.671\right.$, $p<0.001)$, absolute $\mathrm{HRr}$ at 2-minutes post-exercise $\left[\mathrm{F}_{1,37}=12.125, \mathrm{p}=0.001, \mathrm{~d}=1.12(0.43,1.78)\right]$, relative $\mathrm{HRr}$ at 2-minutes post-exercise $\left[F_{1,37}=10.845, p=0.002, d=1.06(0.37,1.71)\right]$, absolute $\mathrm{HRr}$ at 5 -minutes post-exercise $\left[F_{1,37}=6.418, p=0.016, d=0.81(0.14,1.45)\right]$, and relative $H R r$ at 5 -minutes post-exercise $\left[F_{1,37}=5.888, p=0.020\right.$, $d=0.77(0.10,1.41)]$. Absolute and relative HRr after the maximal GXT (Figure 1) showed a similar rate of recovery during the three minutes between short- and long-term recoveries. Effect sizes for these specific differences are all considered large. It is noteworthy that the untrained group's mean $\mathrm{VO}_{2}$ max is classified as poor to very poor regardless of age and gender to indicate that this truly was an untrained group (Riebe et al., 2017). Additionally, the untrained group had significantly slower HRr than the trained group at both time points, indicating a higher risk of all-cause mortality.

Table 1. Participant demographics and baseline study metrics

\begin{tabular}{|c|c|c|c|}
\hline Metric & Trained & Untrained & Effect Size $(95 \% \mathrm{Cl})$ \\
\hline Age (years) & $31.5 \pm 14.7$ & $30.1 \pm 12.4$ & $0.10(-0.53,0.73)$ \\
\hline Height $(\mathrm{cm})$ & $172.1 \pm 10.8$ & $170.0 \pm 11.6$ & $0.19(-0.45,0.82)$ \\
\hline Mass $(\mathrm{kg})^{*}$ & $70.6 \pm 11.6$ & $103.9 \pm 29.5$ & $-1.53(-2.21,-0.79)$ \\
\hline$B M l^{*}$ & $23.7 \pm 2.6$ & $36.0 \pm 8.0$ & $-2.14(-2.88,-1.31)$ \\
\hline Body Fat Percent $(\%)^{*}$ & $22.0 \pm 7.1$ & $41.3 \pm 8.1$ & $-2.55(-3.33,-1.66)$ \\
\hline Heart Rate: Rest ${ }^{*}$ & $67.9 \pm 13.2$ & $83.7 \pm 11.6$ & $-1.27(-1.93,-0.55)$ \\
\hline Systolic BP (mmHg) & $120.1 \pm 12.9$ & $126.4 \pm 14.7$ & $-0.46(-1.09,-0.19)$ \\
\hline Diastolic BP (mmHg) & $76.5 \pm 8.6$ & $78.9 \pm 9.4$ & $-0.38(-1.00,0.27)$ \\
\hline $\mathrm{VO}_{2} \max \left(I \cdot \mathrm{min}^{-1}\right)$ & $3.10 \pm 0.80$ & $2.65 \pm 0.90$ & $0.53(-0.12,1.16)$ \\
\hline $\mathrm{VO}_{2} \max \left(\mathrm{ml} \cdot \mathrm{kg}^{-1} \cdot \mathrm{min}^{-1}\right)^{*}$ & $42.3 \pm 6.8$ & $26.1 \pm 8.8$ & $2.08(1.26,2.81)$ \\
\hline Max heart rate $\left(b \cdot \mathrm{min}^{-1}\right)$ & $185.3 \pm 14.4$ & $180.7 \pm 13.6$ & $0.33(-0.31,0.95)$ \\
\hline POMS Score: Vigor, session $1^{*}$ & $-16.9 \pm 5.8$ & $-12.3 \pm 5.0$ & $0.83(0.15,1.46)$ \\
\hline Mean HR, session $2\left(b \cdot \min ^{-1}\right)$ & $134.8 \pm 10.5$ & $136.0 \pm 6.3$ & $-0.14(-0.76,0.50)$ \\
\hline Peak HR, session $2\left(b \cdot \mathrm{min}^{-1}\right)$ & $157.3 \pm 10.9$ & $154.5 \pm 12.4$ & $0.24(-0.40,0.87)$ \\
\hline POMS Score: Vigor, session $2^{*}$ & $-16.3 \pm 6.8$ & $-11.0 \pm 5.8$ & $0.83(0.16,1.47)$ \\
\hline
\end{tabular}

"Denotes significant group differences $(p<0.05)$.

For the second testing session (Figure 2), significant group differences were found for absolute $\mathrm{HRr}$ at 1 -minute post-exercise $\left[F_{1,37}=8.017, p=0.007, d=0.91(0.23,1.55)\right]$, relative $H R r$ at 1-minute post-exercise $\left[F_{1,37}=9.92\right.$, $p=0.003, d=1.03(0.34,1.68)]$, absolute $H R r$ at 2-minutes post-exercise $\left[F_{1,37}=17.035, p<0.001, d=1.33(0.61\right.$, 
2.00)], relative $\mathrm{HRr}$ at 2-minutes post-exercise $\left[F_{1,37}=21.049, \mathrm{p}<0.001, d=1.48(0.74,2.16)\right]$, absolute $\mathrm{HRr}$ at 5 -minutes post-exercise $\left[F_{1,37}=20.604, p<0.001, d=1.46(0.72,2.13)\right]$, and relative $\mathrm{HRr}$ at 5 -minutes post-exercise $\left[F_{1,37}=26.210, p<0.001, d=1.65(0.89,2.34)\right]$, and vigor as measured by the POMS $\left(F_{1,37}=6.908, p=0.012\right)$. The effect sizes for the group differences in $\mathrm{HRr}$ are considered large.

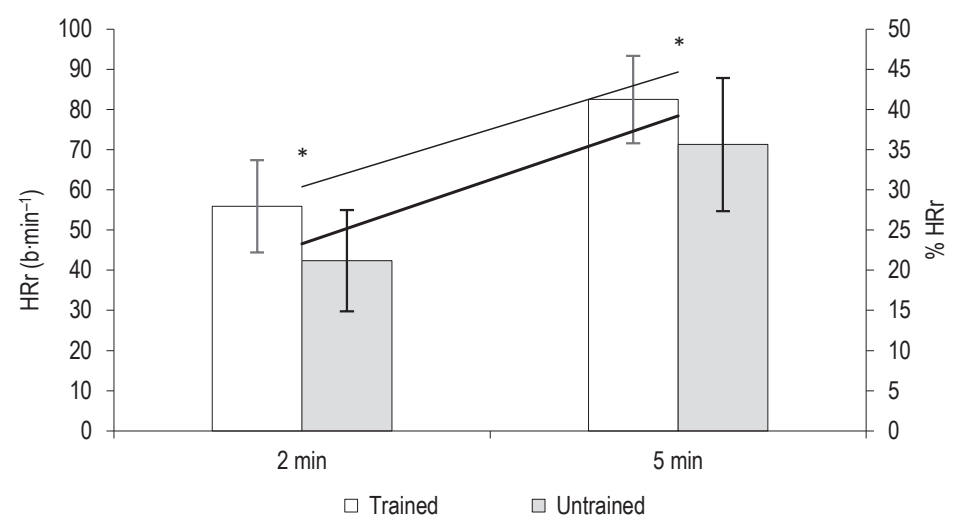

"Denotes a statistically significant difference between groups $(p<0.05)$.

Figure 1. Absolute HRr (primary y-axis) and relative HRr (secondary y-axis) 2-min and 5-min after the participants completed a maximal GXT

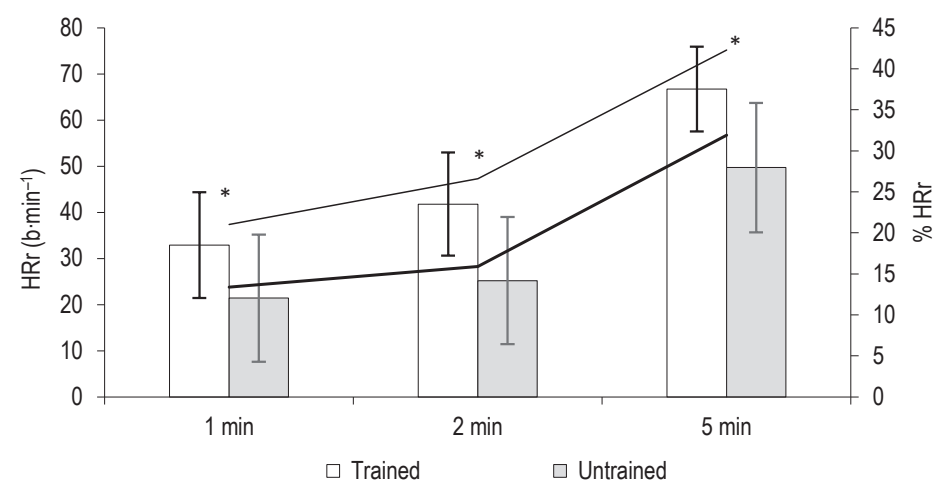

${ }^{*}$ Denotes a statistically significant difference between groups $(p<0.05)$.

Figure 2. Absolute HRr (primary y-axis) and relative HRr (secondary y-axis) 1-min, 2-min, and 5-min after the participants completed a 20-minute moderate-intensity treadmill exercise 


\section{Regression analyses}

The regression analyses revealed significant predictors of $\mathrm{HRr}$ for both groups (Table 2). Resting HR was a significant primary predictor for short-term $\mathrm{HRr}$ for the trained group. Peak HR, primarily for short-term recovery, and mean HR, only after submaximal recovery, also showed to be significant predictors for this group. Interestingly, none of the variables were statistically significant for the 1-min $\mathrm{HRr}$ after the submaximal exercise. For the untrained group, resting HR was also the primary variable for predicting HRr, but other variables such as peak HR (mostly in long-term recovery), $\mathrm{VO}_{2}$ max, mean $\mathrm{HR}$, age, and body fat (all significant with recovery after submaximal exercise) were also significant predictors. $\mathrm{HRr}$ was not significantly related to POMS for either group at any time point. Of particular note is that R-values tended to be higher in the untrained group than the trained group, indicating greater predictive value and influence of the physiological variables on $\mathrm{HRr}$.

Tahle 2. Predictive variables for heart rate recovery after maximal and submaximal exercise

\begin{tabular}{|c|c|c|c|c|c|c|c|c|}
\hline & Resting HR & Peak HR & $\mathrm{VO}_{2} \max$ & Mean HR & Age & $\% \mathrm{BF}$ & Trained & Untrained \\
\hline \multicolumn{9}{|c|}{ Maximal Exercise } \\
\hline Abs 2-min & ${ }^{*} \dagger$ & & & & & & $R=0.551, p=0.010$ & $R=0.799, p<0.001$ \\
\hline Rel 2-min & ${ }^{*} \dagger$ & & & & & & $R=0.609, p=0.003$ & $R=0.764, p<0.001$ \\
\hline Abs 5-min & $\dagger$ & $\dagger$ & & & & & $\mathrm{N} / \mathrm{A}$ & $R=0.809, p<0.001$ \\
\hline Rel 5-min & $\dagger$ & * & & & & & $R=0.447, p=0.042$ & $R=0.661, p=.003$ \\
\hline \multicolumn{9}{|c|}{ Submaximal Exercise } \\
\hline Abs 1-min & $\dagger$ & & $\dagger$ & $\dagger$ & & & $\mathrm{N} / \mathrm{A}$ & $R=0.967, p<0.001$ \\
\hline Rel 1-min & $\dagger$ & & $\dagger$ & $\dagger$ & & & $\mathrm{N} / \mathrm{A}$ & $R=0.977, p<0.001$ \\
\hline Abs 2-min & ${ }^{*} \dagger$ & & $\dagger$ & $\dagger$ & & & $R=0.583, p=0.006$ & $R=0.959, p<0.001$ \\
\hline Rel 2-min & * & ${ }^{*} \dagger$ & & ${ }^{*} \dagger$ & & $\dagger$ & $R=0.818, p<0.001$ & $R=0.927, p<0.001$ \\
\hline Abs 5-min & $\dagger$ & ${ }^{*} \dagger$ & & & $\dagger$ & & $R=0.591, p=0.005$ & $R=0.903, p<0.001$ \\
\hline Rel 5-min & $\dagger$ & & & & $\dagger$ & & $\mathrm{N} / \mathrm{A}$ & $R=0.811, p<0.001$ \\
\hline
\end{tabular}

* Indicates significant predictors for the trained group, and $\dagger$ indicates significant predictors for the untrained group.

\section{Disculssion}

The trained group had a significantly faster absolute and relative $\mathrm{HRr}$ and a higher relative $\mathrm{VO}_{2}$ max than the untrained group. This was demonstrated both in the maximal testing session, as well as the submaximal exercise session, and these findings are consistent previous research (Borresen, Lambert, 2008; Brinkworth et al., 2006; Brown, Brown, 2007; Bunn et al., 2017; Carroll et al., 2012; Dixon, Kamath, McCartney, Fallen, 1992; Lamberts, Swart, Capostagno, Noakes, Lambert, 2010; Sugawara, Murakami, Maeda, Kuno, Matsuda, 2001). Interestingly, the graphical representations of the absolute and relative $\mathrm{HRr}$ show that the gap in recovery between the two groups tends to be the same regardless of the time point (Figures 1 and 2). This indicates that the two groups have the same slope and pattern of recovery. The trained group recovered faster within a shorter time frame after exercise than the untrained group, but the untrained group was able to keep up with this "recovery pace" during the longer recovery period. These results agree with data previously published by Pierpont et al. (2013).

Results also showed a difference in resting HR and no difference in peak HR between groups. Resting HR was expected to be lowered in trained individuals, indicating a greater parasympathetic tone and CV efficiency. 
This further demonstrates the significant improvements that can be made to resting HR and HRr with regular CV training (Georgiopoulou et al., 2012; Matsuo et al., 2014; Thomson et al., 2010). No difference between groups for peak HR indicates that both groups worked at approximately the same intensity during both exercise sessions, which is important to address with recovery. The POMS score for vigor is the last group difference of note. The higher score for the trained group indicated higher robustness, stamina, and a more positive outlook. In theory, this outlook could indirectly influence autonomic control on the heart, but the multiple regression analysis from the present study does not support this concept. This suggests that, while different between groups, mental state had no effect on $\mathrm{HRr}$. This result does not agree with research among competitive athletic teams that emphasizes mental health status being a significant predictor of performance and recovery (Crust, Clough, 2005; Kuan, Roy, 2007).

Detailed analysis of the trained group revealed that resting $\mathrm{HR}$ was the most common predictor of $\mathrm{HRr}$ shortly after (2-min) exercise. Resting HR is primarily controlled by intrinsic factors and parasympathetic influence (Daanen, Lamberts, Kallen, Jin, Van Meeteren, 2012), and trained individuals tend to have a stronger vagal tone than untrained. Thus the correlations between short-term HRr and resting HR for this population shows dominance of the parasympathetic nervous system very quickly post-exercise (Coote, 2010). Long-term HRr (5-min) was linked with maximal $\mathrm{HR}$, providing a greater indication that intensity and duration of the exercise is more influential on this longer portion of recovery (Hautala et al., 2001; Murrell et al., 2007). The untrained group showed similar trends in that resting $\mathrm{HR}$ and maximal $\mathrm{HR}$ were strong predictors of both short- and long-term $\mathrm{HRr}$ respectively. Other factors like $\mathrm{VO}_{2}$ max, mean $\mathrm{HR}$, age, and body fat showed to be predictors of $\mathrm{HRr}$ for this group after submaximal exercise. There have been mixed results in the literature with these factors. A previous study showed no correlation between $\mathrm{VO}_{2}$ max and $\mathrm{HRr}$ in recreationally trained individuals (Bunn et al., 2017), but another study showed that $\mathrm{VO}_{2} \mathrm{max}$ and physical activity participation were correlated with $\mathrm{HRr}$ in trained persons, with the stronger predictor of $\mathrm{HRr}$ being physical activity participation (Lee, Mendoza, 2012). Collectively these data suggest that perhaps after one reaches at least a threshold of "average" $\mathrm{VO}_{2}$ max, then level of fitness is no longer a significant predictor for $\mathrm{HRr}$. More research on this hypothesis needs done to prove the merit of this concept.

As a direct comparison between those persons of higher body mass and lower body mass, this study makes some assumptions about the level of physical fitness in each group. This could be identified as a limitation in study design, as the groups may not be truly different in their health status as determined by mass and BMI alone; though the statistical difference between groups and large effect sizes help to mitigate those assumptions. Another limitation may be that assessment of psychological variables was completed only prior to activity participation. This single assessment before each session does not assess any potential changes in participant mental state after completing exercise, and may contribute to the absence of vigor as a contributing factor to HRr.

Using the data from this study, further assessment of a potential link between $\mathrm{HRr}$ and psychological state of the participant is useful, as it still may be a valuable tool when evaluating a patient for total body wellness and fitness and the "before and after" effect of exercise on mental health and physiologic markers of fitness. Additionally, it may be beneficial to further explore if a physical activity participation and/or CV fitness level threshold exists in relation to healthy $\mathrm{HRr}$. Diseased and disabled populations could use this information to reduce their risk for allcause mortality. 


\section{Conclusion}

This study further bolsters the growing body of literature that uses $\mathrm{HRr}$ as a predictive tool for overall health, demonstrating that a group of trained participants has a quicker $\mathrm{HRr}$ after maximal and submaximal exercise relative to an untrained population. It also reinforces the physiologic theory that $\mathrm{HRr}$ is modifiable with regular CV exercise. Yet, it poses additional questions about a threshold in activity participation and fitness for optimal HRr, as well as questions regarding mood status and its relationship to fitness and health, and further exploration of those theories is still needed.

\section{References}

Borresen, J., Lambert, M.I. (2008). Autonomic control of heart rate during and after exercise: measurements and implications for monitoring training status. Sports Medicine, 38 (8), 633-646.

Bourgeois, A., LeUnes, A., Meyers, M. (2010). Full-scale and short-form of the profile of mood states: A factor analytic comparison. Journal of Sport Behavior, 33 (4), 355-376.

Brinkworth, G.D., Noakes, M., Buckley, J.D., Clifton, P.M. (2006). Weight loss improves heart rate recovery in overweight and obese men with features of the metabolic syndrome. American Heart Journal, 152 (4), 693 e691-696. DOI: 10.1016/j.ahj.2006.07.019.

Brown, S.J., Brown, J.A. (2007). Resting and postexercise cardiac autonomic control in trained master athletes. The Journal of Physiolical Sciences, 57 (1), 23-29. DOI: 10.2170/physiolsci.RP012306.

Bunn, J., Manor, J., Wells, E., Catanzarito, B., Kincer, B., Eschbach, C. (2017). Heart rate recovery and the role of cardiovascular fitness in recovery. Journal of Human Sport and Exercise, 12 (2), 349-357.

Carroll, S., Marshall, P., Ingle, L., Borkoles, E. (2012). Cardiorespiratory fitness and heart rate recovery in obese premenopausal women. Scandinavian Journal of Medicine \& Science in Sports, 22 (6), e133-139. DOI: 10.1111/j.1600-0838.2012.01522.x.

Coote, J.H. (2010). Recovery of heart rate following intense dynamic exercise. Experimental Physiology, 95 (3), 431-440. DOI: 10.1113/ expphysiol.2009.047548.

Crust, L., Clough, P.J. (2005). Relationship between mental toughness and physical endurance. Perceptual and Motor Skills, 100 (1), 192-194. DOI: 10.2466/pms.100.1.192-194.

Daanen, H.A., Lamberts, R.P., Kallen, V.L., Jin, A., Van Meeteren, N.L. (2012). A systematic review on heart-rate recovery to monitor changes in training status in athletes. International Journal of Sports Physiology and Performance, 7 (3), 251-260.

Dixon, E.M., Kamath, M.V., McCartney, N., Fallen, E.L. (1992). Neural regulation of heart rate variability in endurance athletes and sedentary controls. Cardiovascular Research, 26 (7), 713-719.

Esco, M.R., Williford, H.N., Olson, M.S. (2011). Skinfold thickness is related to cardiovascular autonomic control as assessed by heart rate variability and heart rate recovery. Journal of Strength and Conditioning Research, 25 (8), 2304-2310. DOI: 10.1519/ JSC.0b013e3181f90174.

Georgiopoulou, V.V., Dimopoulos, S., Sakellariou, D., Papazachou, O., Gerovasili, V., Tasoulis, A., Agapitou, V., Vogiatzis, I., Roussos, C. Nanas, S. (2012). Cardiopulmonary rehabilitation enhances heart rate recovery in patients with COPD. Respirartory Care, 57 (12), 2095-2103. DOI: 10.4187/respcare.01485.

Hautala, A., Tulppo, M.P., Makikallio, T.H., Laukkanen, R., Nissila, S., Huikuri, H.V. (2001). Changes in cardiac autonomic regulation after prolonged maximal exercise. Clinical Physiology, 21 (2), 238-245.

Kazis, L.E., Anderson, J.J., Meenan, R.F. (1989). Effect sizes for interpreting changes in health status. Medical Care, 27 (3 Suppl), S178-189.

Kim, M.K., Tanaka, K., Kim, M.J., Matsuo, T., Tomita, T., Ohkubo, H., Maeda, S., Ajisaka, R. (2010). Epicardial fat tissue: relationship with cardiorespiratory fitness in men. Medicine \& Science in Sports \& Exercise, 42 (3), 463-469. DOI: 10.1249/ MSS.0b013e3181b8b1f0.

Kokkinos, P., Myers, J., Doumas, M., Faselis, C., Pittaras, A., Manolis, A., Kokkinos, J.P., Narayan, P., Papademetriou, V., Fletcher, R. (2012). Heart rate recovery, exercise capacity, and mortality risk in male veterans. European Journal of Preventive Cardiology, 19 (2), 177-184. DOI: 10.1177/1741826711398432.

Kuan, G., Roy, J. (2007). Goal Profiles, Mental Toughness and its Influence on Performance Outcomes among Wushu Athletes. Journal of Sports Science and Medicine, 6 (CSSI-2), 28-33. 
Lamberts, R.P., Swart, J., Capostagno, B., Noakes, T.D., Lambert, M.I. (2010). Heart rate recovery as a guide to monitor fatigue and predict changes in performance parameters. Scandinavian Journal of Medicine \& Science in Sports, 20 (3), 449-457. DOI: 10.1111/j.1600-0838.2009.00977.x.

Lee, C.M., Mendoza, A. (2012). Dissociation of heart rate variability and heart rate recovery in well-trained athletes. European Journal of Applied Physiology, 112 (7), 2757-2766. DOI: 10.1007/s00421-011-2258-8.

Mackenzie, B. (2001). Profile of Mood States (POMS). Rerieved from: https://www.brianmac.co.uk/poms.htm (31.12.2016).

Matsuo, T., Saotome, K., Seino, S., Eto, M., Shimojo, N., Matsushita, A., lemitsu, M., Ohshima, H., Tanaka, K., Mukai, C. (2014). Low-volume, high-intensity, aerobic interval exercise for sedentary adults: VO(2)max, cardiac mass, and heart rate recovery. European Journal of Applied Physiology, 114 (9), 1963-1972. DOI: 10.1007/s00421-014-2917-7.

McNair, D.M. (1971). Manual for the profile of Mood States. San Diego, CA.

Murrell, C., Wilson, L., Cotter, J.D., Lucas, S., Ogoh, S., George, K., Ainslie, P.N. (2007). Alterations in autonomic function and cerebral hemodynamics to orthostatic challenge following a mountain marathon. Journal of Applied Physiology (1985), 103 (1), 88-96. DOI: 10.1152/japplphysiol.01396.2006.

Pierpont, G.L., Adabag, S., Yannopoulos, D. (2013). Pathophysiology of exercise heart rate recovery: a comprehensive analysis. Annals of Noninvasive Electrocardiology, 18 (2), 107-117. DOI: 10.1111/anec.12061.

Pronk, N.P., Crouse, S.F., Rohack, J.J. (1995). Maximal exercise and acute mood response in women. Physiology \& Behavior, 57 (1), $1-4$.

Riebe, D., Ehrman, J., Liguori, G., Magal, M. (2017). ACSM's Guidelines for Exercise Testing and Prescription (10 ed.). Philadelphia: Wolters Kluwer.

Schmikli, S.L., De Vries, W.R., Backx, F.J. (2010). Profile of Mood States is Sensitive to Changes in Workload and Perceived Recovery. Medicine \& Science in Sports \& Exercise, 42, 832-833.

Singh, T.P., Rhodes, J., Gauvreau, K. (2008). Determinants of heart rate recovery following exercise in children. Medicine \& Science in Sports \& Exercise, 40 (4), 601-605. DOI: 10.1249/MSS.0b013e3181621ec4.

Sugawara, J., Murakami, H., Maeda, S., Kuno, S., Matsuda, M. (2001). Change in post-exercise vagal reactivation with exercise training and detraining in young men. European Journal of Applied Physiology, 85 (3-4), 259-263. DOI: 10.1007/s004210100443.

Thomson, R.L., Buckley, J.D., Noakes, M., Clifton, P.M., Norman, R.J., Brinkworth, G.D. (2010). Heart rate recovery improves after weight loss in overweight and obese women with polycystic ovary syndrome. Fertility and Sterilility, 93 (4), 1173-1178. DOI: 10.1016/j.fertnstert.2008.12.003.

Weinberg, R.S., Gould, D. (2011). Foundations of Sport and Exercise Psychology (5th ed.). Champaign, IL: Human Kinetics.

Weir, K. (2011). The exercise effect. Monitor on Psychology, 42 (11), 48-51.

Cite this anticle aS: Bunn, J.A., Wells, E.K., Avery, M.L., Manor, J.P. (2018). Differences in Physiological Influences on Heart Rate Recovery between Trained and Untrained Adults. Central European Journal of Sport Sciences and Medicine, 2 (22), 13-21. DOI: 10.18276/cej.2018.2-02. 